\title{
Peripartum cardiomyopathy in uncorrected tetralogy of fallot: a case report
}

\author{
Mukund Vasantrao Phutane ${ }^{1 *}$, Varsha Ansaram Kale ${ }^{2}$, Vishal Vijayrao Patil ${ }^{1}$, N. O. Bansal ${ }^{1}$
}

\begin{abstract}
${ }^{1}$ Department of Cardiology, The Grant Medical College and Sir J. J. Group of Hospital, Mumbai, Maharashtra, India
${ }^{2}$ Department of Nuclear Medicine, Seth G.S.M.C. and KEM Hospital Mumbai, Maharashtra, India
\end{abstract}

Received: 02 December 2017

Accepted: 30 December 2017

\section{*Correspondence:}

Dr. Mukund Vasantrao Phutane,

E-mail: mak27remo@gmail.com

Copyright: (C) the author(s), publisher and licensee Medip Academy. This is an open-access article distributed under the terms of the Creative Commons Attribution Non-Commercial License, which permits unrestricted non-commercial use, distribution, and reproduction in any medium, provided the original work is properly cited.

\begin{abstract}
Tetralogy of Fallot (ToF) is the most common cyanotic congenital heart with incidence about $10 \%$ of all congenital heart disease. Natural survival to the fourth decade is extremely rare (only about 3\%), but there is a tendency of increasing number of women with cyanotic congenital heart disease living 3 to 4 decades and are becoming pregnant. Because of significant physiology adaptation and changes, pregnancy and delivery process are troublesome for most unhealthy women, including those with uncorrected ToF. For ToF patients, it remains an important cause of maternal morbidity (62.5\%), and even mortality (10\%) and has significant effects on fetal outcome. Discussed below a case of pregnancy in a 30 year old woman with uncorrected ToF, dyspnoea class II of New York Heart Association, on 36th week pregnancy with complication of postpartum cardiomyopathy. Through dedicated medical care, patient's condition improvement can be seen. Prognosis of pregnancy in patient with uncorrected ToF is poor without optimal obstetrical and medical management.
\end{abstract}

Keywords: Uncorrected tetralogy of fallot, Pregnancy, Postpartum cardiomyopathy (PPCM)

\section{INTRODUCTION}

Tetralogy of fallot is the most common cyanotic congenital heart disease, with incidence about $10 \%$ of all congenital heart disease. The defects found in patient with $\mathrm{ToF}$ are caused by a single developmental defect: an abnormal anterior and cephalad displacement of the infundibular (outflow tract) portion of the inter ventricular septum. Four anomalies arising from this defect are (1) pulmonary stenosis, (2) right ventricular hypertrophy (3) overriding aorta (4) nonrestrictive ventricular septum defect. ${ }^{1}$

To reach adulthood, most patients with ToF will need surgery, either palliative or reparative. However, few patients will present as adults without correction of ToF. Natural survival into the fourth decade is extremely rare (only about 3\%). Hemodynamic changes during pregnancy are profound with plasma volume overload, tachycardia and slight fall in peripheral vascular resistance. Cardiac output has to increase 50\% above baseline during second stage of labour and at time of delivery. ${ }^{2}$

Pregnancy is troublesome for most unhealthy women, including those with uncorrected ToF. Due to fall in peripheral vascular resistance there is increase in right to left shunt, with subsequent increase in cyanosis.

During parturation blood loss may lead hypotension and eventually the right to left shunt. Thus, ToF patient notice a deterioration during pregnancy and parturation. These remain an important cause of maternal morbidity 
$(62.5 \%)$, and mortality $(10 \%)$ and has significant effects on fetal outcome. ${ }^{3}$

\section{CASE REPORT}

A 30 year old woman G1P1L0A0 referred to cardiology department at 36 weeks and 2 days of gestation. She suffered from some congenital heart disease since childhood and was advised to undergo repair but could not do so, due to financial reason. Now she presented with dyspnoea NYHA class II symptom. Her pulse was $80 /$ minute, blood pressure $112 / 70 \mathrm{~mm}$ of mercury, respiratory rate $-20 /$ minute, temperature $98.4^{\circ} \mathrm{F}$, cyanosis with grade II clubbing of finger, with oxygen saturation of $89 \%$ at hospitalization. On auscultation S1 was normal with single S2 and loud ejection systolic murmur over pulmonary area with normal respiratory examination.

Laboratory examination showed hemoglobin $13 \mathrm{~g} / \mathrm{dL}$, hematocrit $62 \%$, WBC 10,200/dl, platelet count 2,20,000/cc, Other blood chemistry, urine analysis, and serum electrolytes were within normal limit. ECG showed sinus rhythm, QRS rate 78/minute, right QRS axis deviation 90, normal PR interval, incomplete right bundle branch block, and no significant ST- T changes.

Echocardiogram (Figure 1) showed levocardia and hypertrophy of inter ventricular septum and right ventricle, large VSD, bidirectional shunt, overriding aorta $>50 \%$, severe. Pulmonary stenosis, fair PA size, left aortic arch, no coarctation of the aorta, no collateral from descendent aorta with LV ejection fraction $60 \%$ finding consisting with Tetralogy of Fallot.

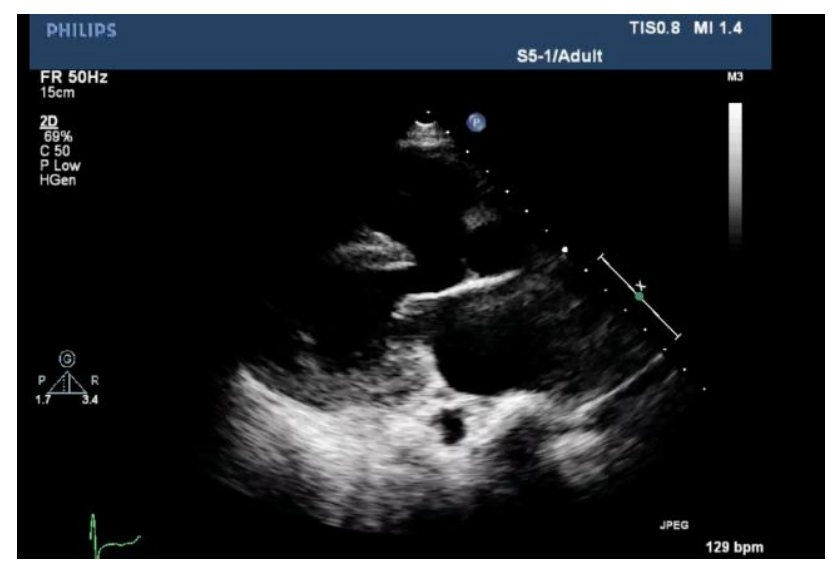

Figure 1: Two-dimensional echocardiogram showing large sub aortic ventricular septal defect with over riding of aorta and right ventricular hypertrophy.

We preferred vaginal delivery over caesarian section for delivery mode. Multidisciplinary team, including anesthesiologists, cardiologists, cardio thoracic surgeons, obstetrics and pediatricians, managed the patient. Foetal examination was normal. She was kept in propped up position with continuous $\mathrm{O} 2$ inhalation. IV fluid and propranolol $20 \mathrm{mg}$ tablet $\mathrm{BD}$ was given. Antibiotic prophylaxis with amoxicillin was administered before episiotomy with cut short of second stage of labour. Delivery was carried out without complications and was uneventful. The baby weighted $2200 \mathrm{~g}$ and was stable. During labour and postpartum period we monitored the mother with blood pressure, heart rate, oxygen saturation and temperature three times daily. After delivery her general condition was fair. Uterus was well contracted. Her postnatal period was uneventful. She forced hospital to discharge with assurance of further follow up in cardiology OPD and counseled regarding need of cardiac surgery. Risk of future pregnancy explained and advised a barrier method for contraception.

She lost follow up and came after 1 month with dyspnoea NYHA class III, investigations showed hemoglobin $13.5 \mathrm{~g} / \mathrm{dL}$, hematocrit $60.8 \%$, WBC $6200 / \mathrm{cc}$, platelet count $2.80,000 / \mathrm{cc}$. Arterial blood gas analysis showed pH: 7.307, pCO2: $24.3 \mathrm{mmHg}, \mathrm{pO} 2: 50.6 \mathrm{mmHg}$, saturation of $\mathrm{O} 2$ : $87 \%$, HCO3-: $12.6 \mathrm{mEq} / \mathrm{l}$; showing uncompensated metabolic acidosis, urine analysis, and serum electrolytes were within normal limit. She was afebrile, her pulse rate was 128/minute, blood pressure $100 / 70 \mathrm{~mm}$ of mercury, respiratory rate $28 /$ minute, bilaterally symmetric chest movement with crepitation on right lower zone, and baseline cyanosis. On auscultation S1 was normal, single S2, gallop rhythm and ejection systolic murmur over pulmonary area.

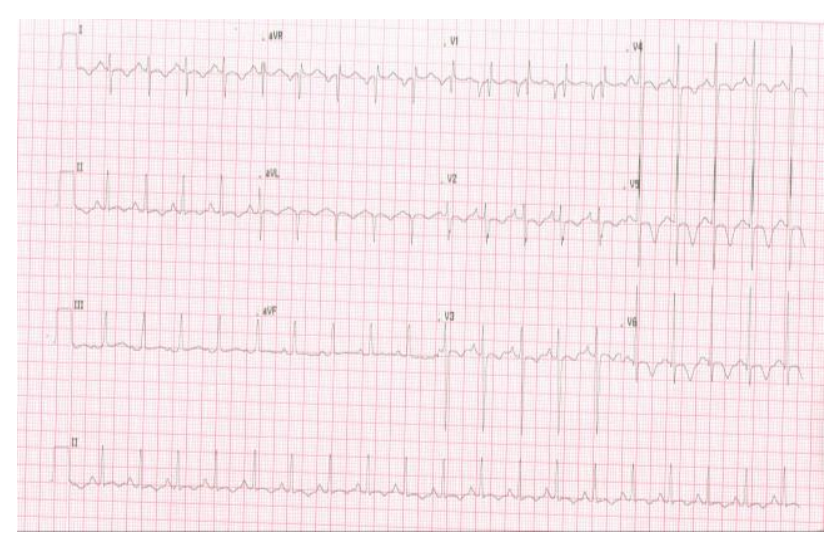

Figure 2: ECG showing sinus tachycardia, right QRS axis deviation 90, left atrial enlargement with normal

PR interval, incomplete right bundle branch block, and $T$ wave inversion in lead $I$, aVL, V2 to V6.

ECG (Figure 2) showed sinus tachycardia, QRS rate 114/minute, right QRS axis deviation 90, left atrial enlargement with normal PR interval, incomplete right bundle branch block, and $\mathrm{T}$ wave inversion in lead $\mathrm{I}$, aVL, V2 to V6.

Echocardiography this time showed congested IVC, TOF physiology but there was striking finding with LV ejection fraction $<35 \%$. Serial Trop I was markedly positive with decreasing trend, with significant ECG findings, and $\mathrm{X}$ ray we came to conclusion of TOF with postpartum cardiomyopathy. We stabilized patient with 
diuretics. There is very small and controversial data regarding long-term management of uncorrected TOF patient with postpartum cardiomyopathy. We advised our patient diuretics and after stabilization low dose of ACE inhibitor and $\mathrm{B}$ blocker with close clinical and echocardiography follow up. Counseled about their cardiovascular risk and advised against pregnancy.

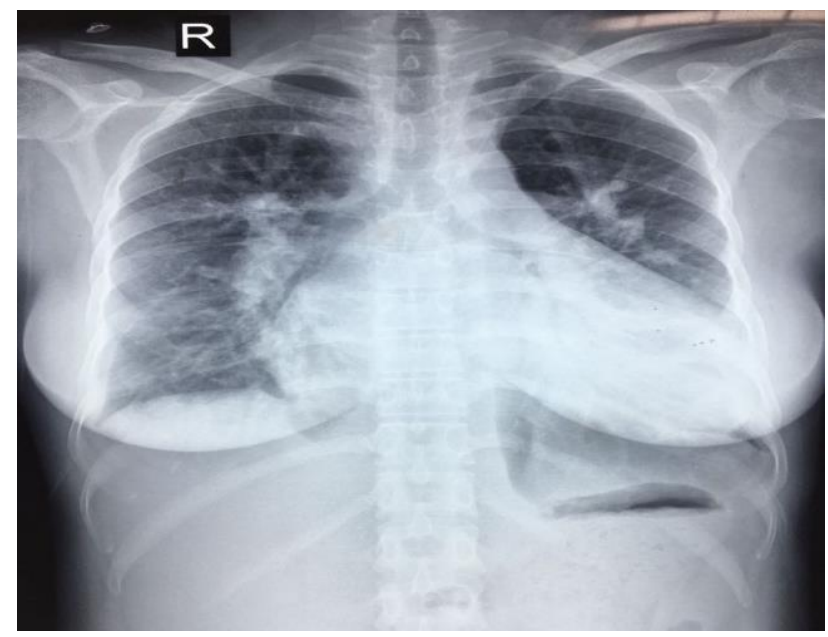

Figure 3: X ray Chest PA view showing cardiomegaly.

\section{DISCUSSION}

The main characteristic of TOF is cyanosis. In TOF, cyanosis usually results from RV outflow obstruction with inadequate pulmonary blood flow and right-to-left shunt at the level of ventricles. ${ }^{4}$

Prognosis of pregnant women with TOF depends upon whether their pathology corrected or not, either palliative or definitive surgery or they have residual defects post procedure. Woman with TOF during pregnancy are more likely to decompensate because they are not able to meet the additional demands. A state of hypotension or volume overload is poorly tolerated and specially become vulnerable to decompensation during the later months of pregnancy and postpartum. Patients with TOF have an increased risk of fetal loss, and their babies are more likely to have congenital anomalies. ${ }^{5}$

For management of ToF, there is no literature which recommends repairing process of cardiac anomalies during pregnancy. Therefore, the only way to overcome this problem is by managing the pregnancy medically. Pregnancy in uncorrected TOF can proceed with total bed rest and supportive care, and is classified as high risk pregnancy. These patients needed to be monitored throughout the pregnancy, labor, and post parturition. Foetus need to be monitored for growth and well-being. The most important prognostic factor for maternal and fetal outcomes is oxygen saturation. ${ }^{6}$

In first phase of delivery, it is recommended to avoid the usage of uterotonic agents, some suggest the use of low dose epidural analgesia for reducing oxygen consumption. Many literatures prefer vaginal birth to caesarian section for delivery mode in woman with ToF. In second phase of delivery, forceps usage is recommended. Caesarian section is indicated only for obstetrical indications the reasons is bleeding, that will be lesser if vaginal birth mode is chosen over caesarian section. If caesarian section is indicated, general anesthesia is chosen to avoid hypotensive effect of regional anesthesia.

Administration fluid before procedure is recommended to avoid hypotension. In third phase of delivery, it is recommended to limit the bleeding amount. These patients should be given prophylactic antibiotic because they are at high risk of infective endocarditis. ${ }^{7}$

The pregnancy with uncorrected TOF carries major risks of maternal morbidity, including heart failure, arrhythmia, endocarditis, thromboembolism, even maternal mortality. In present case, patient presented with postpartum cardiomyopathy (PPCM). There is strongly suggested a role for vascular dysfunction, hormonal insults, and underlying genetics in PPCM. LV ejection fraction at presentation best predicts rate of recovery of PPCM.

The risk of PPCM relapse in patients with persistent LV dysfunction is much higher in subsequent pregnancy than in those who have normalized LV function. Most patients will recover by 6 months, with little change in EF thereafter. Treatment is focused, as with other forms of systolic failure, on controlling volume status, neutralizing maladaptive neurohormonal responses, and preventing thromboembolic and arrhythmic complications. Neurohormonal blockade with ACE inhibitors or angiotensin receptor blockers can be used postpartum. ${ }^{8}$

Education about reparative procedure after pregnancy and contraception method to prevent further pregnancy before reparative process is needed to be given. The number of TOF patients are steadily increasing each year so the number of TOF patients with pregnancy. There is a strong need for carefully coordinated multidisciplinary efforts to manage these patients and maintain long-term follow-up.

\section{CONCLUSION}

The management of such patients with proper monitoring, bed rest, beta blocker, diuretics and ACE inhibitor significant improvement can be seen. Corrective surgery after stabilization should be done. Without proper cardiac care and obstetrical management, prognosis of pregnancy in patient with uncorrected ToF is poor.

Postpartum these patients should be vigilantly monitored both clinically and by ECG, X ray, echocardiography for postpartum cardiomyopathy like complications. Such patients should be counselled regarding their 
cardiovascular risk and advised against further pregnancy. Further studies are required for management of such patients.

Funding: No funding sources Conflict of interest: None declared

Ethical approval: Not required

\section{REFERENCES}

1. Sinto R, Nasution SA. Pregnancy in a Woman with Uncorrected Tetralogy of Fallot. Acta Med Indones. 2009 Apr; 41(2):81-6.

2. Pandey K, Arya S, Sharma D, Sharma CM. Pregnancy with uncorrected tetralogy of Fallot: a case report. Int J Reprod Contracept Obstet Gynecol 2013;2:677-9.

3. Silvia P, Alberto D, Enza D, Silvia M, Marta D, Guido M. Pregnancy and maternal uncorrected tetralogy of Fallot: A case report and review of the literature. J Cases Obstet Gynecol. 2015;2(3):43-7
4. Siu SC, Colman JM. Heart disease and pregnancy. Heart. 2001;85:710-5.

5. Veldtman GR, Connolly HM, Grogan M, Ammash NM, Warnes CA. Outcomes of pregnancy in women with tetralogy of fallot. J Am Coll Cardiol. 2004;44:174-80.

6. Warnes CA. Pregnancy and heart disease. Braunwalds Heart disease: a textbook of cardiovascular medicine. $10^{\text {th }}$ ed. Philadelphia, PA: Elsevier/Saunders;2015:1755.

7. Gupta K, Bajaj B, Das B. Successful outcome of pregnancy in uncorrected tetralogy of fallot. Int $\mathbf{J}$ Reprod Contracept Obstet Gynecol. 2014 Sep;3(3):799-802.

8. Arany Z, Elkayam U. Peripartum Cardiomyopathy. Circulation. 2016;133:1397-1409.

Cite this article as: Phutane MV, Kale VA, Patil VV, Bansal NO. Peripartum cardiomyopathy in uncorrected tetralogy of fallot: a case report. Int J Reprod Contracept Obstet Gynecol 2018;7:748-51. 